\title{
PROGRESS IN DISRUPTION PREVENTION FOR ITER
}

\author{
E.J. Strait ${ }^{1}$, J.L. Barr ${ }^{1}$, M. Baruzzo ${ }^{2}$, J.W. Berkery ${ }^{3}$, R.J. Buttery ${ }^{1}$, P.C. de Vries ${ }^{4}$, N.W. Eidietis ${ }^{1}$, R.S. Granetz ${ }^{5}$,
} J.M. Hanson ${ }^{3}$, C.T. Holcomb ${ }^{6}$, D.A. Humphreys ${ }^{1}$, J.H. Kim ${ }^{7}$, E. Kolemen ${ }^{8}$, M. Kong ${ }^{9}$, M.J. Lanctot ${ }^{1 *}$, M. Lehnen $^{4}$, E. Lerche ${ }^{10,11}$, N.C. Logan ${ }^{8}$, M. Maraschek ${ }^{12}$, M. Okabayashi ${ }^{8}$, J.K. Park ${ }^{8}$, A. Pau ${ }^{13}$, G. Pautasso ${ }^{12}$, F.M. Poli ${ }^{8}$, C. Rea ${ }^{5}$, S.A. Sabbagh ${ }^{3}$, O. Sauter ${ }^{9}$, E. Schuster ${ }^{14}$, U.A. Sheikh ${ }^{9}$, C. Sozzi ${ }^{15}$, F. Turco ${ }^{3}$, A.D. Turnbull $^{1}$, Z.R. Wang ${ }^{8}$, W.P. Wehner ${ }^{14}$, L. Zeng ${ }^{16}$

${ }^{1}$ General Atomics, PO Box 85608, San Diego, CA 92186-5608, USA

${ }^{2}$ Consorzio RFX, corso Stati Uniti 4, 35127 Padova, Italy

${ }^{3}$ Columbia University, New York, NY 10027, USA

${ }^{4}$ ITER Organization, Route de Vinon sur Verdon, 13067 St Paul Lez Durance, France

${ }^{5}$ Plasma Science and Fusion Center, Massachusetts Institute of Technology, Cambridge, MA, USA

${ }^{6}$ Lawrence Livermore National Laboratory, Livermore, CA 94551, USA

${ }^{7}$ National Fusion Research Institute, Daejon, Republic of Korea

${ }^{8}$ Princeton Plasma Physics Laboratory, Princeton, NJ 08543, USA

9́École Polytechnique Fédérale de Lausanne (EPFL), Swiss Plasma Center (SPC), CH-1015 Lausanne, Switzerland

${ }^{10}$ CCFE Fusion Association, Culham Science Centre, Abingdon, United Kingdom

${ }^{11}$ LPP-ERM/KMS, Association EUROFUSION-Belgian State, TEC partner, Brussels, Belgium

${ }^{12}$ Max-Planck-Institute für Plasma Physik, D-85748, Garching, Germany

${ }^{13}$ Electrical and Electronic Engineering Department, University of Cagliari, 09123 Cagliari, Italy

${ }^{14}$ Lehigh University, Bethlehem, PA 18015-3085, USA

${ }^{15}$ Istituto di Fisica del Plasma-Universita degli Studi di Milano-Bicocca, Milan, Italy

${ }^{16}$ Institute of Plasma Physics, Chinese Academy of Sciences, Hefei 230031, People's Republic of China

"Present address: U.S. Department of Energy, Washington, DC, USA.

\begin{abstract}
Key plasma physics and real-time control elements needed for robustly stable operation of high fusion power discharges in ITER have been demonstrated in recent research worldwide. Recent analysis has identified the current density profile as the main drive for disruptive instabilities in discharges simulating ITER's baseline scenario with high and low external torque. Ongoing development of model-based profile control and active control of magnetohydrodynamic instabilities is improving the stability of multiple scenarios. Significant advances have been made toward real-time physicsbased prediction of instabilities, including path-oriented analysis, active sensing, and machine learning techniques for prediction that are beginning to go beyond simple disruption mitigation trigger applications. Active intervention contributes to prevention of disruptions, including forced rotation of magnetic islands to prevent wall locking, and localized heating/current drive to shrink the islands. Stable discharge rampdowns have been achieved with the fastest ITER-like scaled current ramp rates, while maintaining an X-point configuration. These elements are being integrated into stable operating scenarios and new event-handling systems for off-normal events in order to develop the physics basis and techniques for robust control in ITER.
\end{abstract}

\section{INTRODUCTION}

The large thermal and magnetic energy contained in a full-performance discharge means that ITER must have an exceedingly low rate of disruptions by the time it reaches DT operation. A disruption budget [1] will be part of the ITER's operating plan [2], in order to track the cumulative effects of all disruptions. Although early operation at reduced parameters will allow more leeway for disruptions, unmitigated disruptions at currents above 8.4 MA may be severe enough (Category III) to be tolerable only once or twice in the machine's lifetime [3]. Thus, as experiments advance toward the planned current of $15 \mathrm{MA}$, the tolerance for unmitigated disruptions is expected to become of the order 1 in $10^{4}$ discharges. Fig. 1 shows one possible scenario for the evolution of ITER's disruption rate [1]. After an initial learning curve at reduced parameters, by the time full fusion power operation is reached the allowable disruption rate per discharge is only $1 \%$, and those few disruptions must be mitigated with $95-99 \%$ accuracy. That is, nearly disruption-free operation will be required, in addition to highly reliable mitigation of any disruptions that do occur [4]. Uncertainties in prediction [5] and mitigation [6] of runaway electrons in ITER disruptions may place an even higher premium on disruption-free operation.

Sustaining a low disruptivity tokamak plasma is fundamentally a plasma control problem. Beginning with a minimally disruptive target scenario, continuously-operating algorithms in the plasma control system must be 
able to regulate the plasma state to maintain the desired operating point, with passive stability to as many potentially disruptive modes as possible. Any remaining potentially disruptive modes must be actively and robustly stabilized. In addition to quantifiably robust continuous algorithms, the control system must be able to detect and respond asynchronously to hardware faults and off-normal plasma conditions ("exceptions" in ITER terminology) [7], [8] in such a way as to prevent the exception from leading to a disruption. However, the plant and the nominal scenario should be so reliable that exceptions are extremely rare. Disruption mitigation must also be integrated into the plasma control system [9], but is outside the scope of this paper. In ITER, disruption mitigation should be a rarely-used last resort.

This paper provides an overview of recent research toward the goal of disruption-free operation. Section 2 discusses progress in identifying stable operating scenarios and robust control to maintain the desired operating state. Section 3 describes real-time algorithms that detect off-normal conditions, or predict that an instability will occur later in the discharge. Section 4 describes active intervention by various means to enable recovery of stable operation in the same scenario or an alternate one (maximizing the physics productivity of the discharge), or allow a controlled termination of the discharge. These elements contribute to an integrated control solution, capable of robustly maintaining stable plasma conditions, or recognizing and responding to off-normal and fault events so as to reliably prevent disruptions (section 5).

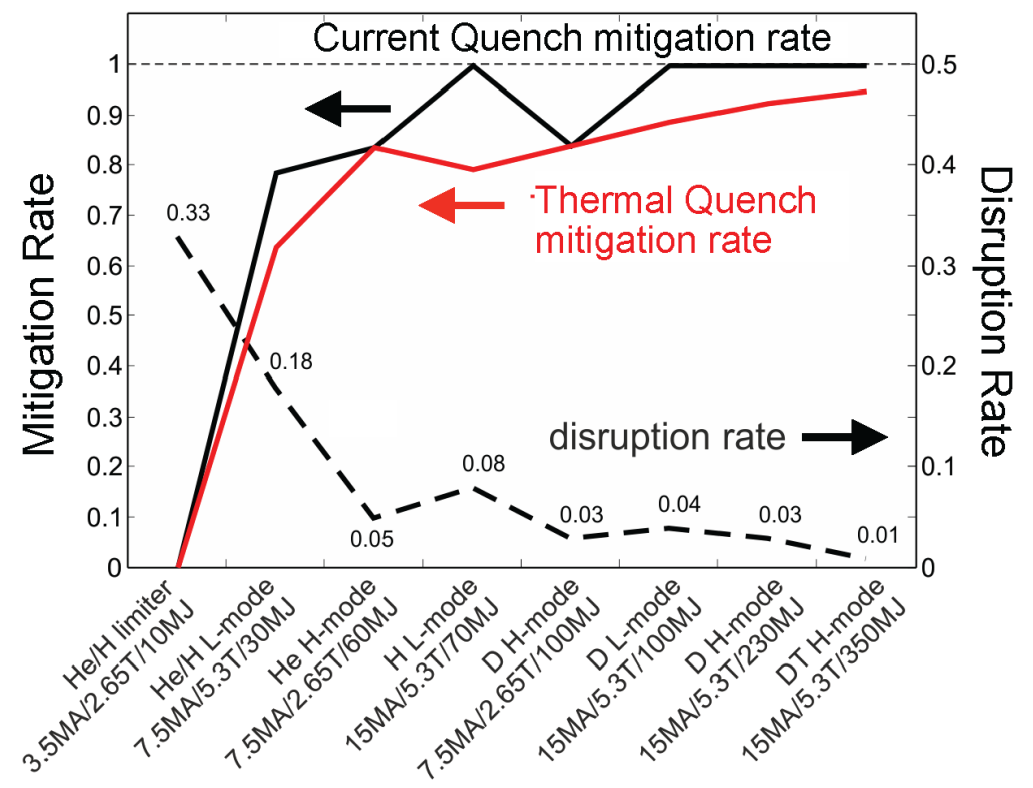

Fig. 1. A potential scenario for ITER's allowable disruption rate and required mitigation rates, over the evolution of the planned research. [Reprinted from M. Lehnen, et al., Proc. 26th IAEA Fusion Energy Conference (Kyoto, 2016), IAEA, Vienna (2017), paper EX/P6-39.]

\section{PLASMA CONFIGURATION CONTROL}

Reliable operation of ITER (and burning plasmas beyond ITER) requires a robustly stable, stationary operating state. Active control measures, up to and including a rapid shutdown by massive impurity injection, must be available to recover from off-normal conditions and prevent a disruption, or mitigate the severity of a disruption. However, it is clear that high reliability is needed in the normal operating state, so that the use of these safeguards is very rare. This is achieved through the selection of a stable plasma configuration, controls to achieve and maintain that configuration, stable paths to access the configuration at the beginning of the discharge and to exit from it at the end, and (where needed) active stabilization to extend the stability limits.

Optimization of the equilibrium configuration has been shown to improve the stability of zero torque ITER-like discharges. Previously, many such discharges in DIII-D disrupted due to tearing modes, and in general neoclassical tearing mode (NTM) stability appeared to become poorer as neutral beam torque was reduced [10]. In JET, NTMs were the largest single cause of disruption with the carbon wall [35], and could enhance core accumulation of impurities in operation with the ITER-like wall [11]. Recent DIII-D results have shown that tearing instabilities in ITER baseline scenario plasmas are related to the shape of the current density profile around the q=2 surface. A deeper current "well" between the ohmic current in the core and the bootstrap current at the edge is correlated with greater instability [12] (see Fig. 2). 


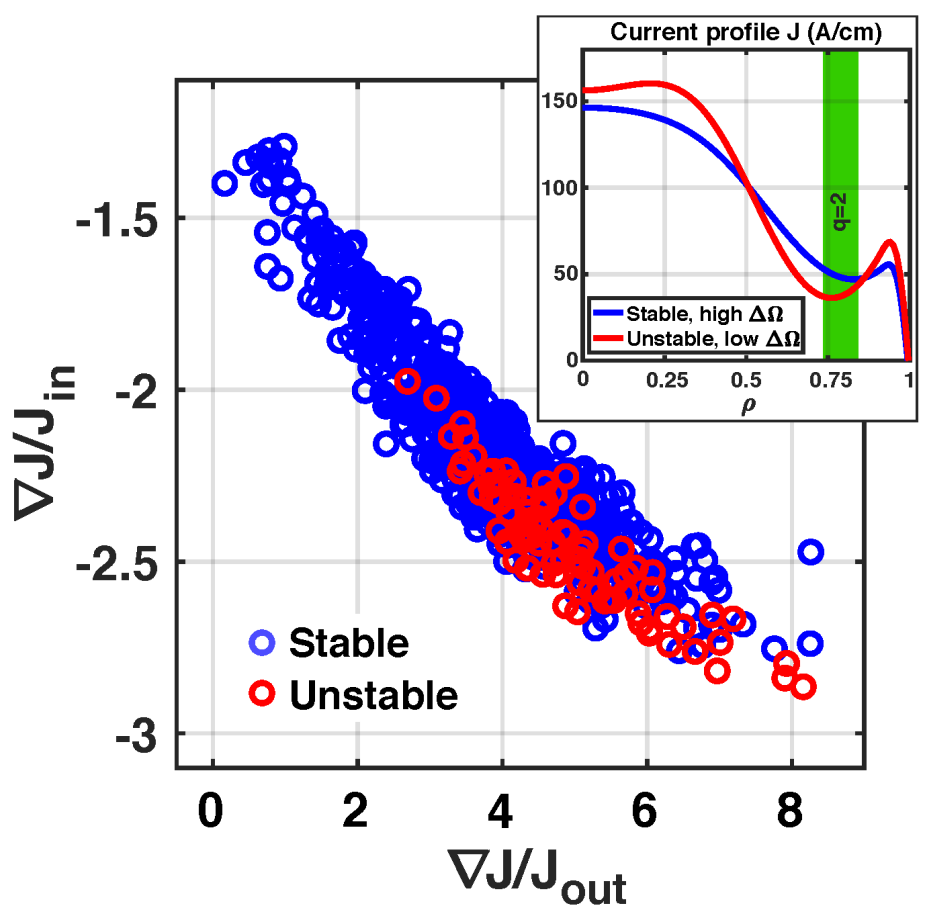

Fig. 2. Current density gradient inside and outside the minimum or "well" of current density near the q=2 surface at $\rho \sim 0.76-0.81$ (see inset) in DIII-D discharges simulating the ITER baseline scenario. Colours indicate discharges stable (blue) or unstable (red) to $\mathrm{m} / \mathrm{n}=2 / 1$ tearing modes. [Reprinted from F. Turco, et al., Nuclear Fusion 58 (2018) 106043]

In addition to the existence of a stable operating state, it is essential to have controls that will maintain the discharge in that state. Tokamak plasma control is complex, with nonlinear, coupled processes to be controlled, and possible bifurcations of the plasma state, putting a premium on integrated, model-based control [13]. The advent of transport simulations that can operate faster than real time [14] will be important for discharge monitoring and control. Simultaneous control of plasma pressure and safety factor using model-based control has been tested in a common environment [85] in TCV using several different controllers, [86], [88], [95], [15]. In DIII-D, as shown in Fig. 3, a model-based feedforward+feedback algorithm [16] controls both neutral beams and gyrotrons to achieve simultaneous targets of plasma energy and a current density profile with high $\mathrm{q}_{\min }$, in development of discharges for non-inductive operation [17]. Model-based control may be applicable to other scenarios as well.
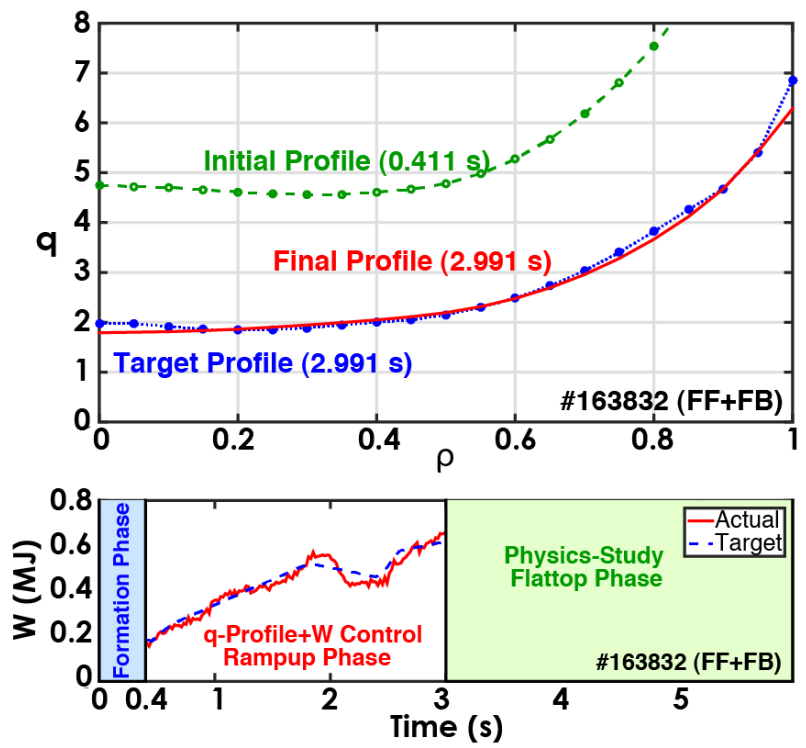

Fig. 3. Demonstration in DIII-D of a feedforward+feedback q-profile control scheme with model-based optimization, achieving a target profile with qmin $=1.9$ while tracking a target value for plasma energy W. [Reprinted from W.P. Wehner, et al., paper EX/P6-39, Proc. th IAEA Fusion Energy Conference (Ahmedabad, 2018), IAEA, Vienna (2019).] 
Impurities can lead to instabilities and disruptions by altering the evolution of the pressure and current density profiles. In the initial operation of JET with an ITER-like metallic wall, disruptions were often caused by high impurity radiation from the plasma core, causing broadening of the current density profile that led to tearing

| modes [82], [96]. Disruptivity is reduced by using central electron heating with ion cyclotron resonance heating (ICRH) to improve the central power balance and reduce core impurity accumulation [82], perhaps similar to the use of central electron cyclotron resonance heating (ECRH) in ASDEX Upgrade with an all-tungsten wall [83]. The JET disruption mitigation system is now in routine use, in many cases triggered by the onset of a locked tearing mode [84].

Maintaining a stable operating state may include extending the bounds of stable operation through direct control of instabilities. Real time active tracking and control of NTMs is well established, but recent analysis [18] indicates that the presence of ITER's blanket modules can cause $\mathrm{m} / \mathrm{n}=2 / 1$ tearing modes to lock in a time significantly shorter than previous estimates that considered only the vacuum vessel wall. If active NTM stabilization is required, this result reduces the time available for re-aiming gyrotrons to suppress the mode after detection, and increases the attractiveness of continuous electyron cyclotron current drive (ECCD) applied at the rational surface to maintain NTM stability. The estimated power requirement of about $5 \mathrm{MW}$ for pre-emptive stabilization of the $2 / 1$ mode is consistent with ITER's electron cyclotron system, and would still allow demonstration of a fusion gain of $\mathrm{Q}=10$ [19]. Small-amplitude sweeping of the deposition location across the rational surface can reduce the requirements for accuracy of aiming, as first tested on TCV [20], [75] and later on ASDEX Upgrade [21]. Alternatively, a recently demonstrated fast resonant diplexer [22] could allow some gyrotrons to switch as needed between central heating/current drive and NTM control, without delay.

A need for control of the sawtooth instability is also anticipated in ITER, in order to avoid long period sawteeth with large amplitude crashes that could trigger an NTM instability [23], and several schemes using ECCD or ion cyclotron range of frequency (ICRF) heating have been proposed [24]. Controlled sawteeth and edge-localized modes (ELMs) can also improve disruptivity by flushing impurities out of the core and edge, respectively. Recent JET experiments have shown that the sawtooth period can be controlled with low field side ICRF heating (as will be available in ITER) near the $\mathrm{q}=1$ surface [25], and that sawtooth pacing can be achieved with modulated central ion cyclotron heating [26], a method that is less sensitive to the deposition location. Fig. 4 shows an example where irregular sawteeth repeatedly seed an $n=2$ tearing mode, but pacing at a higher rate using modulated central ICRH reduces the sawtooth period and eliminates the seeding. Further work is needed to validate ICRF sawtooth control in ITER-relevant high-confinement (H-mode) discharges.
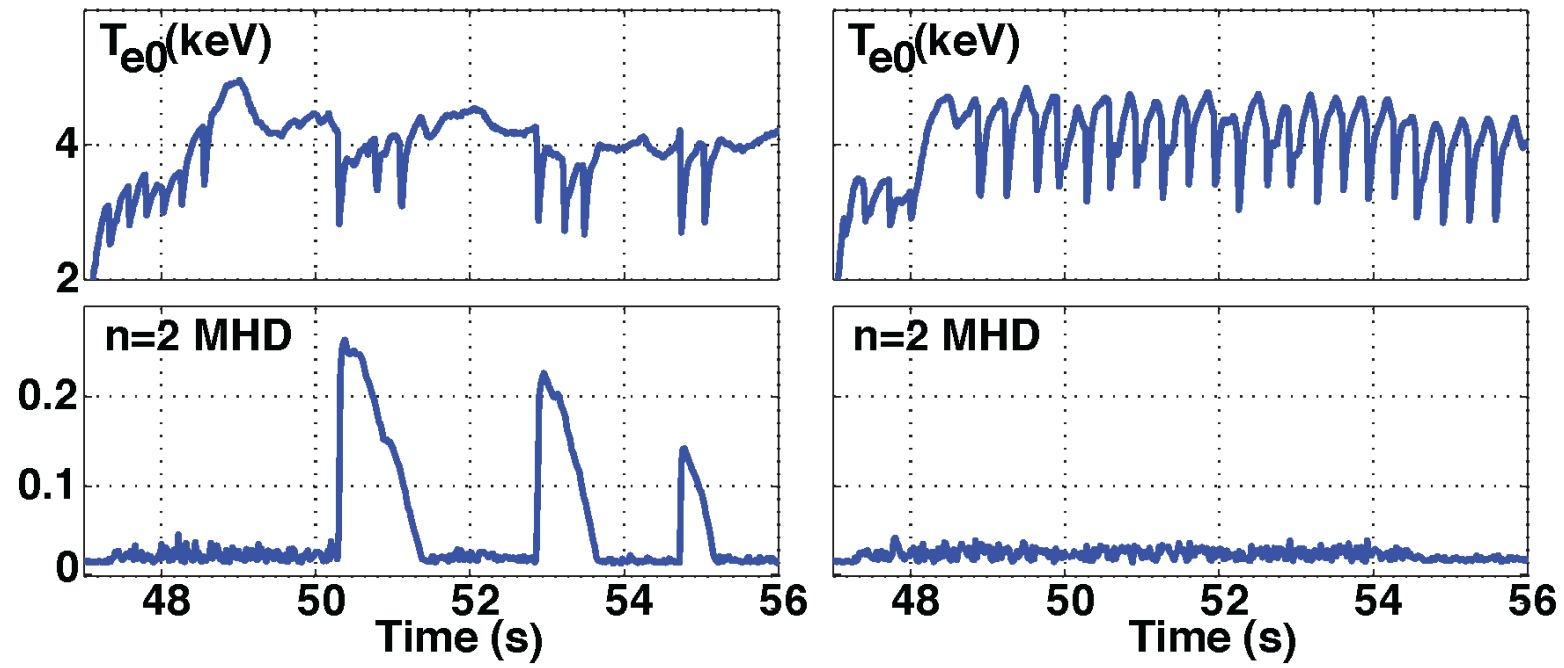

Fig. 4. Comparison of two H-mode discharges in JET with constant (left) and $3 \mathrm{~Hz}$ modulated (right) ICRF heating, illustrating the reliable sawtooth pacing and total suppression of the $n=2$ MHD activity in the latter. Upper panels show ICRH power; lower panels show $n=2$ MHD mode amplitude. [Reprinted from E. Lerche, et al., Nuclear Fusion 57 (2017) 036027.]

Equilibrium control for stable operation includes non-axisymmetric aspects of the equilibrium. A large body of literature exists on the topic of $n=1$ error fields, and ITER will include external non-axisymmetric coils for $n=1$ error field compensation. However, as seen in Fig. 5, recent data from DIII-D and EAST show that the threshold for $n=2$ error field penetration and driven reconnection may be comparable to that of $n=1$ error fields [27]. This 
result suggests that the use of the in-vessel coils for $n=2$ error field correction should be kept as an option for ITER, since the external correction will be hard-wired for $n=1$. More complete data are needed to characterize the scaling of thresholds for $\mathrm{n}=2$ error field penetration in existing devices and to establish criteria for $\mathrm{n}=2$ error field control in ITER.

High beta, high $\mathrm{q}_{\min }$ discharges in ITER's steady state scenario are expected to exceed the no-wall ideal magnetohydrodynamic (MHD) kink stability limit. Despite the predicted stabilizing effects of particle-orbit resonances, active feedback control of non-axisymmetric fields for stabilization of the resistive wall mode or dynamic error field correction may be needed in this regime, in order to avoid instabilities leading to beta collapse and, in some cases, disruption. Experiments on NSTX [28] and DIII-D [29] have shown that feedback stabilization can indeed extend the stable operating range toward the ideal-wall limit (the theoretical maximum). Advanced state space control algorithms can achieve stabilization with external coil arrays [28], [30].
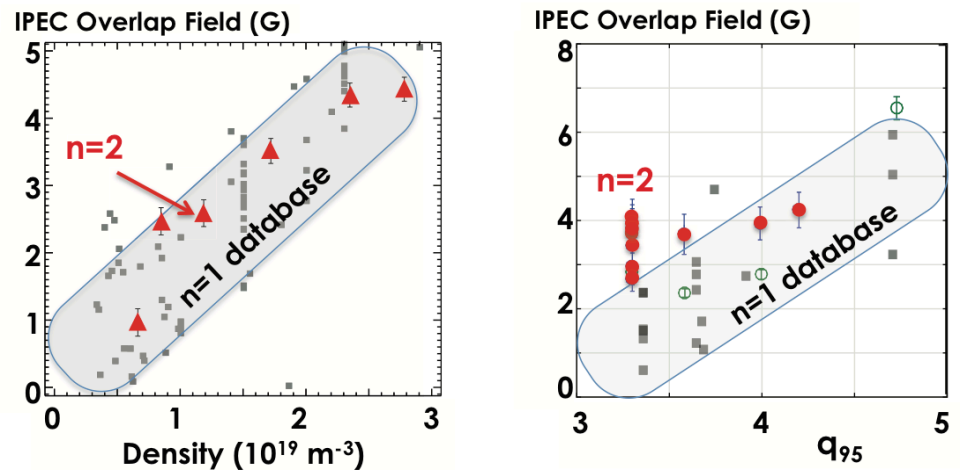

Fig. 5. Density dependence (left panel, DIII-D data) and q95dependence (right panel, EAST data) of the critical "overlap" amplitude for $n=2$ field penetration in Ohmic discharge, compared to $n=1$ thresholds for similar discharges in DIII-D, KSTAR, JET, and (left panel only) NSTX and C-MOD. [Reprinted from M. Lanctot, et al., Nuclear Fusion 57 (2017) 056117.]

\section{REAL-TIME DISRUPTION WARNINGS}

Achieving a low rate of disruptions requires the ability to recognize or anticipate exceptions (i.e., conditions and events that are likely to lead to a disruption) in real time, and to take action that enables a return to normal operation or a controlled shutdown. It is important to distinguish such warnings from those that trigger a rapid shutdown by the disruption mitigation system; the latter require only a short warning time, perhaps as little as $30 \mathrm{~ms}$ before the thermal quench [4], and a binary output. In contrast, in order to allow the discharge to continue, notification of an exception must include information about the nature of the exception, enabling a control decision on a course of action using available actuators, and must occur early enough that the control system has time to change the evolution of the discharge. Potential solutions to this problem include "physicsdriven" methods based on a qualitative or quantitative model of the physical processes that would ultimately lead to an instability, and "data-driven" or machine learning methods, where statistical analysis of a large database of discharges yields empirical correlations between measured data and instabilities. This is an area of rapid development in physics and mathematics.

To date, most real-time systems have used one or more single-parameter physics-based detectors (e.g., MHD mode amplitude, radiated power fraction, global energy confinement level, ...) to trigger a disruption mitigation system when a specified threshold is passed, or (with a lower threshold) to initiate more benign preventive actions. For example, routine JET disruption detection is based on detection of high levels of MHD activity and radiation peaking, precursors to the thermal collapse, as well as large plasma current excursions or voltage spikes, following the thermal spike and signalling the start of the current quench [97]. Analysis of a multimachine database [31] has provided a basis for normalizing the critical locked mode amplitude for disruption, independent of machine size, while analysis of a DIII-D database [32] shows that the proximity of the outer edge of the island to the plasma surface is a key factor in whether a locked mode leads to a disruption. Machinelearning approaches (discussed below) should be applicable to data analysis for optimization of these individual physics-based tests [33] and the control responses. Although each such test is specialized to certain classes of disruptions, multiple threshold tests can be combined with suitable weighting to make a more general warning system with a high success rate [34]. 
Path-oriented analysis seeks to identify specific chains of events that can lead to a disruption [35]. Detection of an event or condition early in the chain could enable a warning signal in time for modification of the discharge before it becomes unstable. The Disruption Event Characterization and Forecasting (DECAF) code [36], now being used to analyze data from several devices, identifies multiple events that may precede a disruption and aims to predict other instabilities using validated physics models such as a reduced model for resistive wall mode stability with kinetic effects [37] or tearing mode torque balance bifurcation to a locked state [38]. In the NSTX discharge shown in Fig. 6, DECAF identifies the onset of a tearing instability and several subsequent events, all of which contribute to a rising disruption warning signal. Multiple variable tests in DECAF have produced early warnings on transport timescales, potentially allowing time for actions to prevent a disruption. Another path-oriented approach is described in [39], including a discussion of the sensor and actuator requirements. Fig. 7 shows an example of this second approach in ASDEX Upgrade of discharge recovery after a warning of crossing the H-mode density limit boundary. As a demonstration, TCV has isolated a specific path to disruption - impurity influx leading to an NTM that later locks - and developed controls to address each step of this path [78].

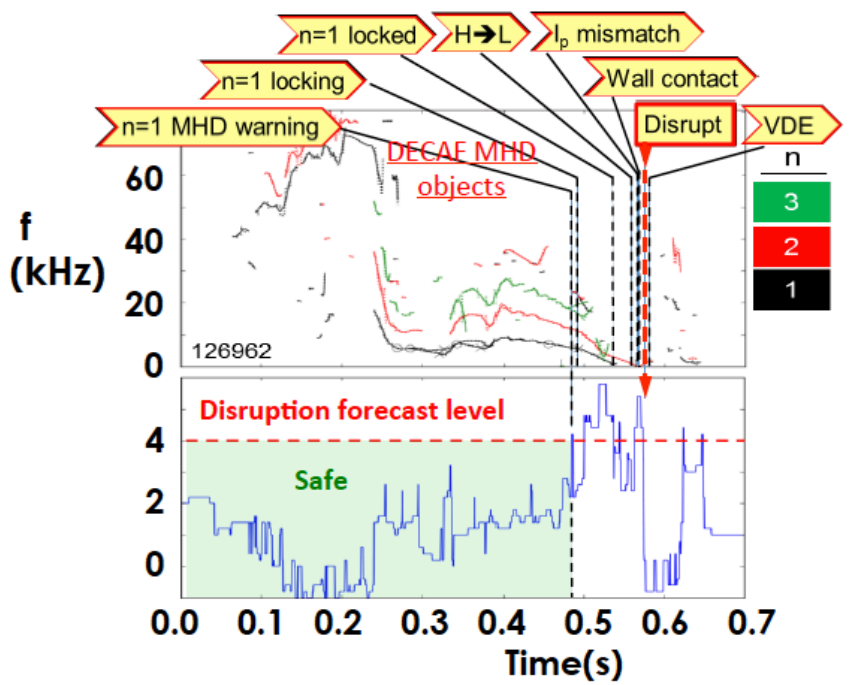

Fig. 6. Upper panel: DECAF decomposition of rotating MHD in an NSTX discharge, with the timing of various events identified by DECAF on the path toward disruption. Lower panel: total MHD warning signal, based on the event chain identified at the top of the figure. [Reprinted from S. Sabbagh, et al., Proc. 27th IAEA Fusion Energy Conference (Ahmedabad, 2018), IAEA, Vienna (2019), paper EX/P6-26.]

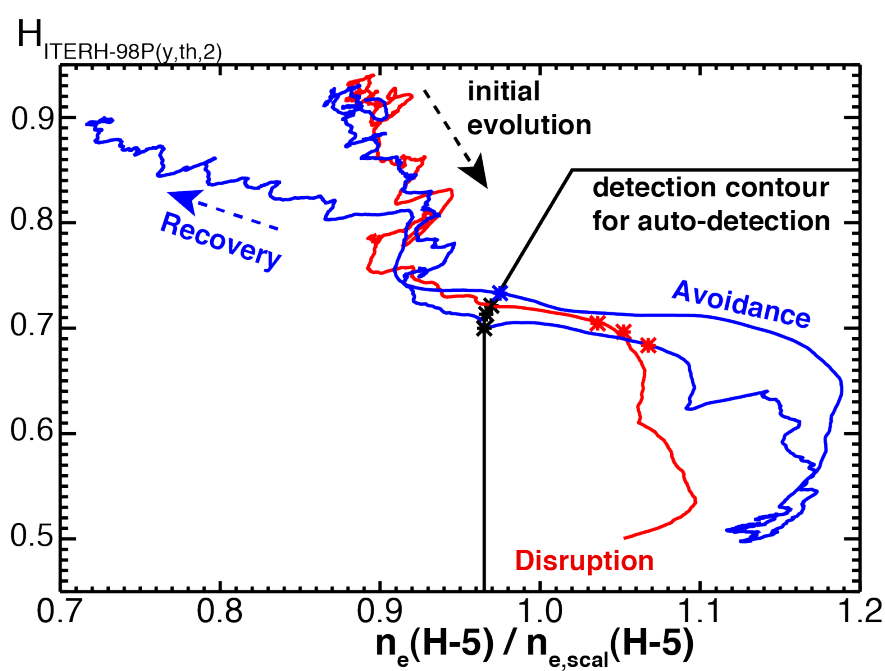

Fig. 7. Operation diagram of H-mode density limit discharges in ASDEX-Upgrade, in terms of scaled energy confinement time vs. scaled density. One discharges disrupts after crossing the density limit detection contour; the other is recovered by turning off gas fueling and applying ECCD. In all cases the proposed trigger threshold would have been early enough to safely avoid the approaching disruption. [Reprinted from M. Maraschek, et al., Plasma Phys. Control. Fusion 60 (2018) 014047.] 
Data driven approaches such as the Advance Predictor of Disruptions (APODIS) system [45] at JET have, so far, been used-tested mainly for binary warnings where the actuator is a disruption mitigation system. Adaptive methods to train an algorithm "from scratch" have achieved satisfactory performance after only a few tens of disruptive shots [46], [47]. Probabilistic predictors [47], [48] express output in terms of a likelihood of disruption rather than a binary classification [47], [48], and the probabilistic approach has also been applied to forecasting the onset of a tearing mode [49]. Cross-machine portability of data-driven algorithms has been a challenge [93]. However, deep-learning algorithms have begun to incorporate time-sequential data and higherdimension data via advanced neural net methods with promising results, including accurate cross-machine predictions [50].

A key challenge for machine learning algorithms is to deliver detailed information about the exception that has been detected, so that the control system can generate an appropriate response to prevent the disruption. Work toward this goal is in progress. As seen in an example from EAST in Fig. 8, Random Forest algorithms can reveal the relative contributions of the various input data signals to the final disruption probability [51], [52]. Generative Topographic Mapping (GTM) reduces a complex multi-dimensional space of input data to a 2dimensional or 3-dimensional space of safe and unstable regions, simplifying the tasks of classification, prediction, and control of instabilities [53], [54] (see Fig. 9). A proposed general approach [55] is to estimate the future trajectory of a discharge in the multidimensional space of input data - or perhaps in a reduced space such as that of Fig. 9 - in order to project its future proximity to disruption boundaries and the time to collision with a boundary; any necessary actions would be based on the gradient of the decision function (e.g. disruption probability) and its dependence on the input data and available actuators. Translating these principles to a practical control scheme is a challenge for future work.

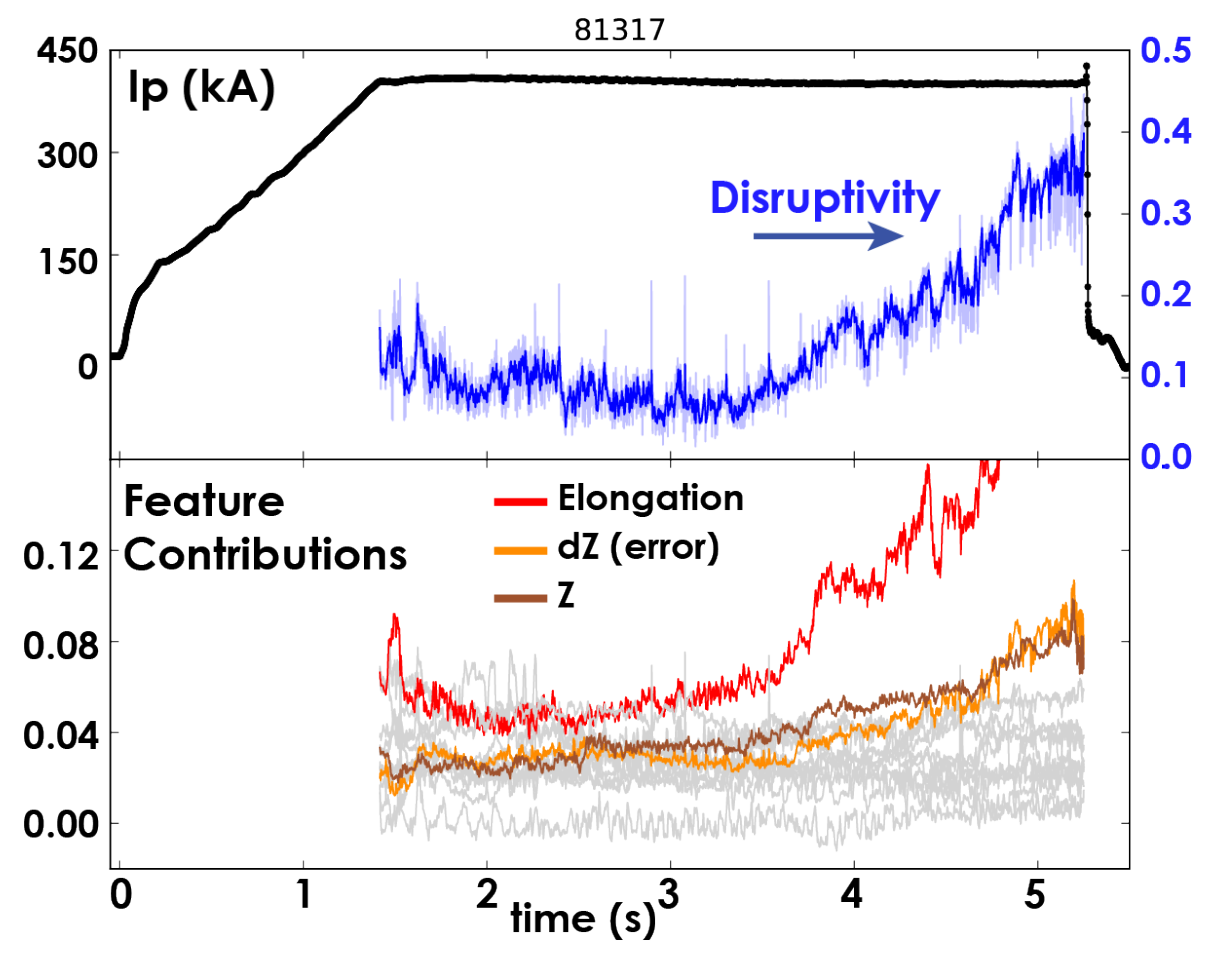

Fig. 8. (a) Disruptivity signal (blue curve) from a Random Forest machine-learning algorithm rises as an EAST discharge approaches a Vertical Displacement Event (VDE) instability. (b) and (c) show the relative importance of the 13 input signals. Rising blue traces indicate high importance for discharge elongation, vertical position, and vertical control error. [Reprinted from R.S. Granetz, et al., Proc. 27th IAEA Fusion Energy Conference (Ahmedabad, 2018), IAEA, Vienna (2019), paper EX/P6-20.] 


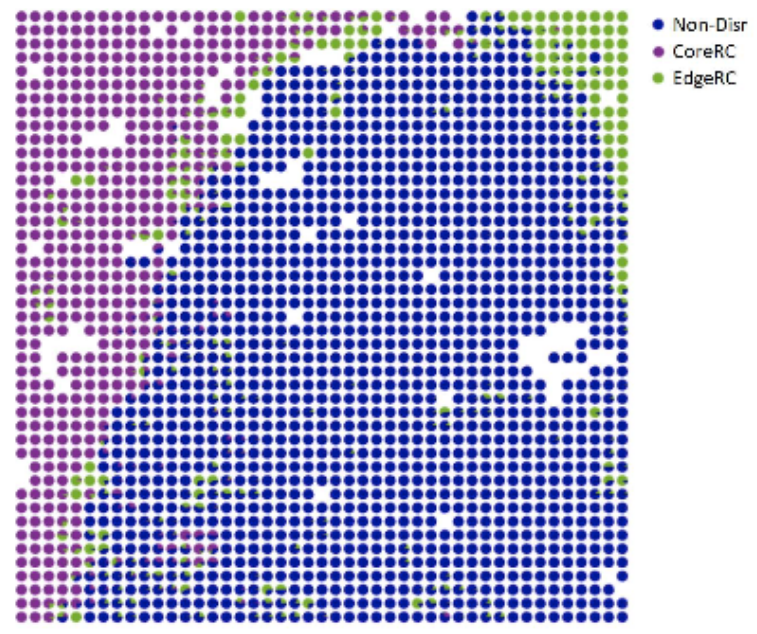

Fig. 9. Generative Topographic Mapping of 5-D JET data (peaking factors of electron temperature, electron density, and radiated power; internal inductance $\ell_{i}$; and $q_{95} / q_{0}$ ratio) to a 2-D "latent space". Colours on the map distinguish stability regimes: core radiative collapse disruptions (CoreRC, magenta), edge radiative collapse (EdgeRC, green), and nondisrupting cases (blue). [Reprinted from A. Pau, et al., IEEE Trans. Plasma Science 46 (2018) 2691.]

Methods for direct assessment of the plasma's stability are conceptually elegant, but are still in their infancy. Recent advances in numerical methods and computing power have enabled first-principles calculation of ideal MHD stability in real time [40] and resistive stability in near real time [41]. Low-frequency active MHD spectroscopy offers the possibility of a direct real-time measurement of plasma stability. Originally developed for measuring the damping rates of ideal MHD modes [42], the technique has also shown a rising response correlated with the onset of tearing modes in ITER baseline scenario discharges in DIII-D [43]. As shown in Fig. 10, the dependence of the inferred damping rate on plasma parameters is in qualitative agreement with ideal MHD modeling [44], and this stability-related measurement has been used for closed-loop control of the heating power in an ITER baseline scenario discharge.
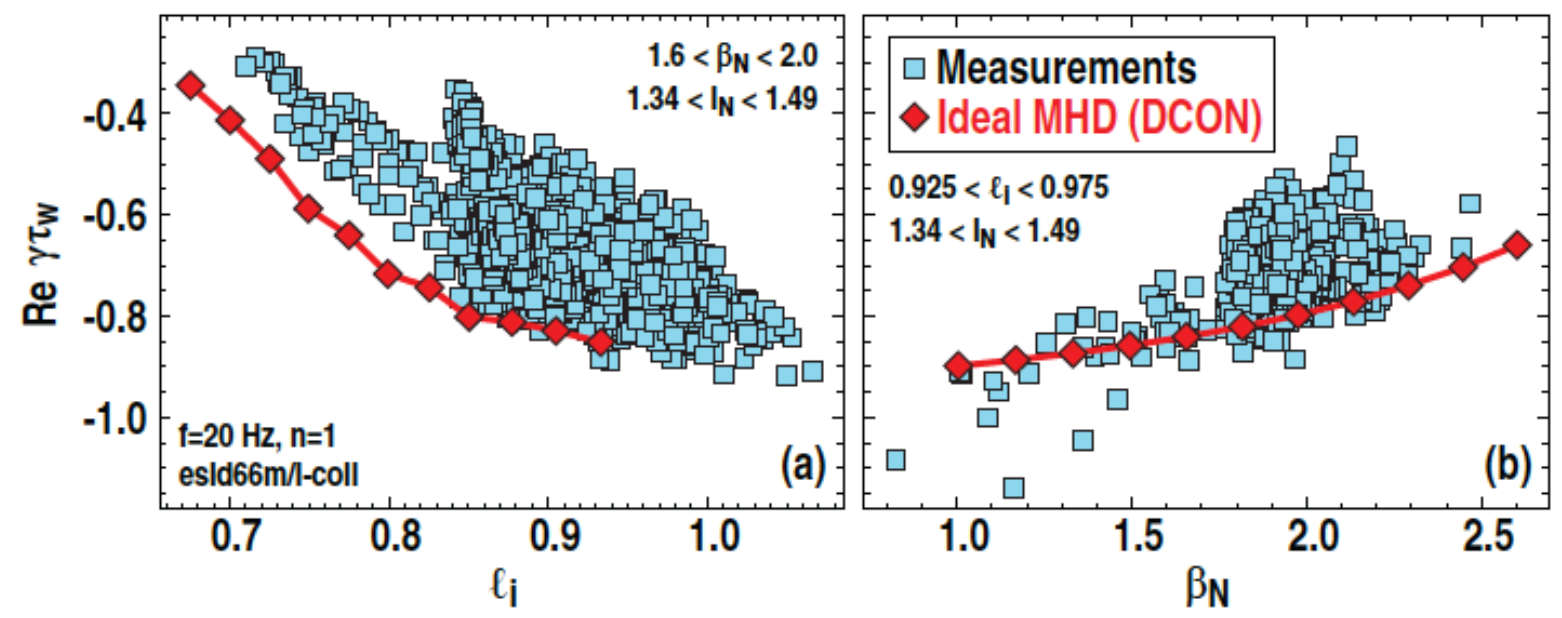

Fig. 10. Stable ITER baseline scenario discharges in DIII-D: (a) $\ell_{i}$ and (b) $\beta_{\mathrm{N}}$ dependences of the normalized RWM growth rate Re(VTw), comparing the values inferred from plasma response measurements (blue squares) with predictions of the linearized, ideal MHD, resistive wall dispersion relation (red diamonds). [Reprinted from J.M. Hanson, et al., Proc. 45th EPS Conf. on Plasma Physics, Europhysics Conf. Abstracts, Vol. 42A (2018) paper P2.1110.]

\section{RESPONSE TO OFF-NORMAL CONDITIONS}

A warning of an off-normal condition that is about to occur, or that is already in progress, is likely to require a response by the control system in order to prevent a disruption, with the response being determined by the nature of the off-normal condition. If feasible, continuing the discharge is preferable to ending it prematurely, and a controlled shutdown is preferable to a rapid termination. For example, the onset of a locked tearing mode is often a precursor to a disruption, but may allow time for other actions aimed at removing the instability and recovering normal operation, or limiting its growth during a controlled shutdown. 
Forced rotation of magnetic islands by applied electromagnetic torque prevents locking to the wall, and reduces the island size. Several experiments [56], [57], [58], have demonstrated entrainment of a locked island by a rotating resonant magnetic perturbation (RMP) at frequencies in the $5-50 \mathrm{~Hz}$ range, limited in frequency by close coupling of the coils to the conducting vacuum vessel wall (Fig. 11). In the absence of strong wall currents, entrainment at several kHz has been achieved [59]. A modulated, non-rotating RMP is also proposed as a means of driving mode rotation [60]. During entrainment, the island maintains a saturated size, and in some cases the H-mode edge pedestal is recovered [61]. Two-fluid modeling shows that island stabilization can occur with sufficient rotation of the RMP relative to the electron fluid [62], [89]. A reduced MHD simulation of the dynamics of island locking and entrainment [63] shows good qualitative agreement with experimental data [90]. A simple 0-D model indicates that in ITER, the internal non-axisymmetric coils could entrain a locked island of 6-8 $\mathrm{cm}$ width at sub-10 $\mathrm{Hz}$ frequencies [58].

Pioneered in FTU, ASDEX Upgrade, and DIII-D, localized injection of electron cyclotron (EC) power at the $\mathrm{q}=2$ surface has been developed as a means of preventing or postponing a disruption after a large amplitude locked mode is present - unlike the pre-emptive or small-amplitude NTM control by ECCD mentioned above in Section 2. Joint experiments in FTU and ASDEX Upgrade show similar behavior in low-beta density limit disruptions and high-beta NTM-driven disruptions [64]. Rutherford equation analysis indicates that while the island is large, electron cyclotron heating plays a larger role in stabilization than does current drive, and is less sensitive to the location of deposition. Real-time mirror steering has been included in the technique [65]. As shown in Fig. 12, TCV has demonstrated control of a large tearing mode destabilized by impurity injection, before or after locking, using ECCD with real-time mirror steering based on real-time equilibrium reconstructions and ray tracing [78].

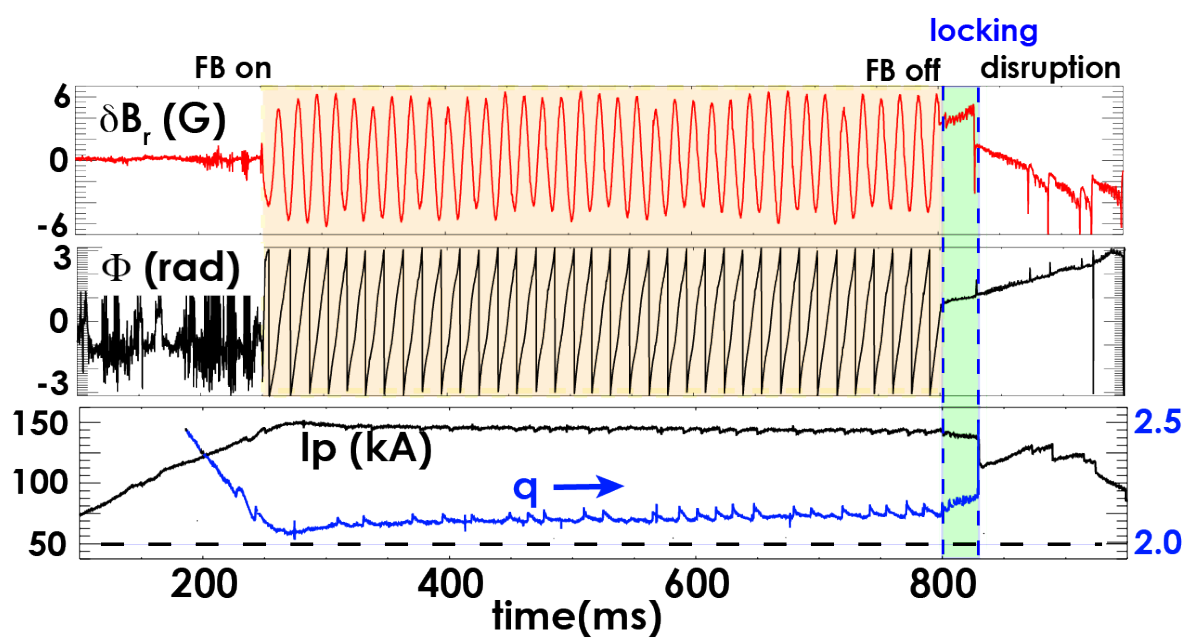

Fig. 11. Long duration of post-unlocked sustainment of $m / n=2 / 1$ mode in RFX-mod (\#33748): (a) 2/1 $\delta B r$ signal, (b) feedback coil current, (c) phase time evolution of the observed 2/1 mode component, (d) safety factor qa and (e) the plasma current. Feedback-controlled magnetic perturbation drives the mode rotation during the orange-shaded interval. After feedback was turned off, the mode survived as a locked mode for a short interval (green) before disruption. [Reprinted from M.Okabayashi, et al., Nuclear Fusion 57 (2017) 016035.] 

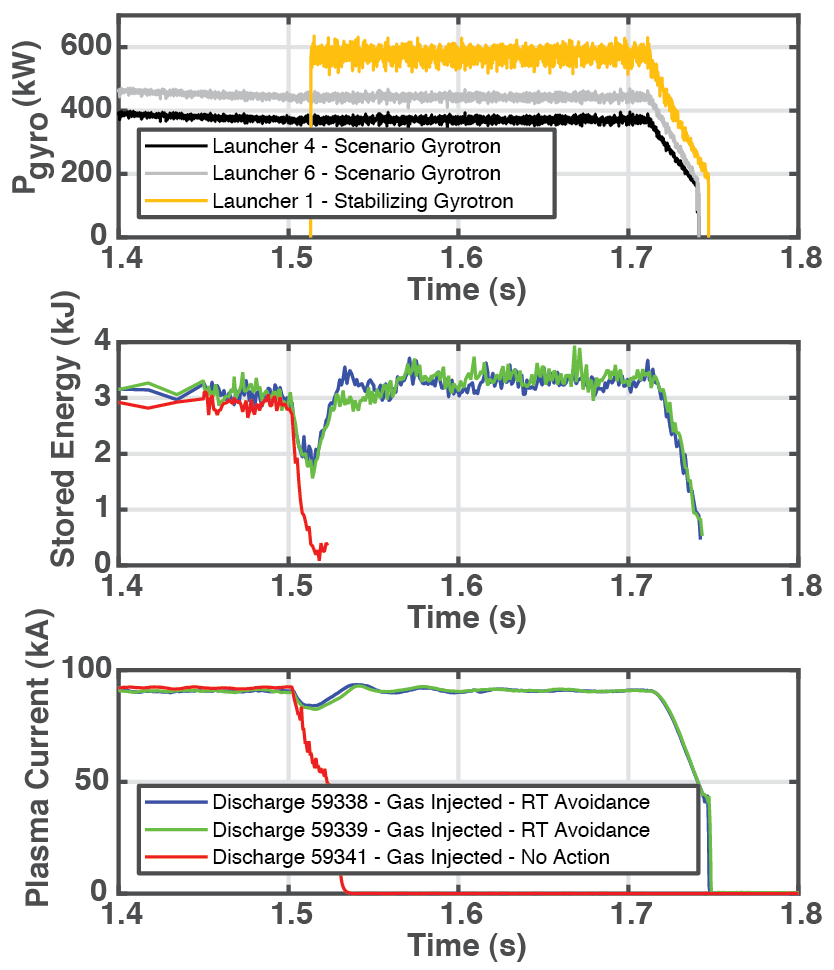

Fig. 12. Gyrotron power, stored energy, and plasma current for TCV discharges with real-time disruption avoidance control. Neon injection at $t=1.5 \mathrm{~s}$ induces a large tearing mode. ECCD targeted at $q=2$, and triggered by SXR and locked mode signals, restores the plasma energy to pre-impurity levels through a combination of mode stabilization and island power balance. Red curves show a reference discharge with no action. [Reprinted from U.A. Sheikh, et al., Nuclear Fusion 58 (2018) 106026.]

Forced island rotation has been combined with injection of EC power into the island for disruption prevention and discharge recovery [66]. A slow, controlled rotation of the island driven by the RMP, with modulated ECCD that is synchronized for deposition in the island O-point, avoids locking while maximizing the efficiency of stabilization by EC power. With this method, suppression of the island and recovery of H-mode operation has been demonstrated.

Stable discharge termination, whether planned or unplanned, is a critical element of disruption-free operation. An extensive study [67] of discharge terminations in existing tokamaks, in comparison with modeling for ITER, shows that ITER's expected trajectory may lie near the edge of the parameter space defined by present experiments. ITER's path is dictated by a rapid reduction of elongation while restricting the increase in internal inductance $\ell_{\mathrm{i}}$, in order to maintain vertical stability. Recent experiments in EAST and DIII-D (Fig. 13) have demonstrated stable rampdowns with equivalent scaled $\mathrm{dI} / \mathrm{dt}$ up to the maximum expected for an unplanned "soft landing" shutdown in ITER, including ITER-like X-point shape with reduced elongation, and low $\ell_{i}$ for vertical stability [68]. KSTAR has also demonstrated a safe rampdown scenario in response to offnormal events, using simplified shape and position controls for robustness [69], and TCV has tested an optimized rampdown trajectory developed from Rapid Plasma Simulator (RAPTOR) simulations [87]. In these scenarios as well as in rampdowns of JET plasmas [70], an important feature is the use of modest auxiliary heating to maintain core power balance during the rampdown, while delaying the high to low confinement $(\mathrm{H}-$ L) transition until the plasma energy has been reduced. JET also utilizes ELM control (vertical kicks and pellet pacing) to minimize impurity accumulation [71]. A multi-machine database is being developed to improve physics-based simulations of the ITER rampdown [91]. 


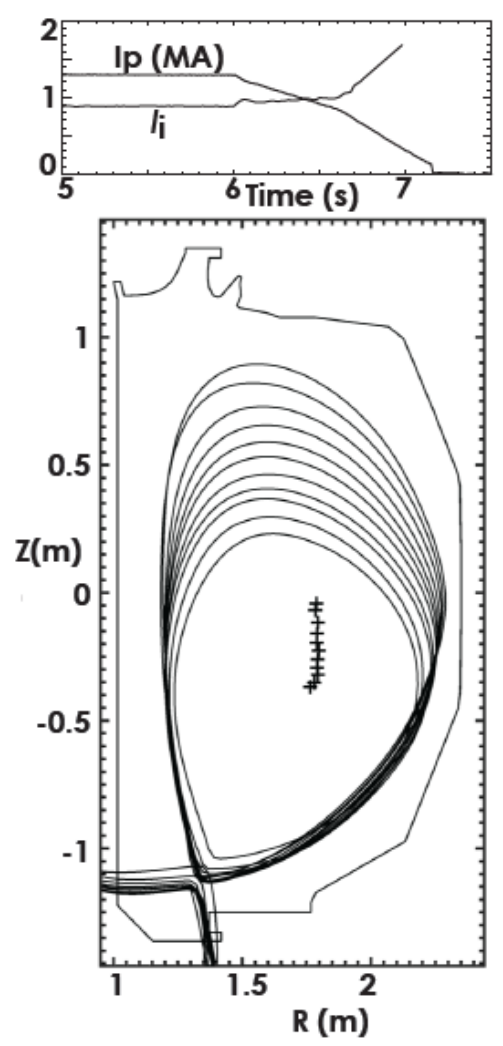

FIG. 13. Stable ITER-like rampdown with X-point configuration, in DIII-D. [Reprinted from J.L. Barr, et al., Proc. 27th IAEA Fusion Energy Conference, IAEA, Vienna (2018), paper EX/P6-21.]

\section{INTEGRATED CONTROL}

The elements needed to minimize the occurrence of disruptions must be integrated, routine, and highly reliable in ITER. These include robustly stable operation, real-time identification of conditions likely to lead to a disruption, and the means to recover, switch to an alternative operating scenario, or terminate the discharge safely after an off-normal event. As discussed above, some building blocks are in hand in present experiments, while others are still under development. Conceptual design of exception handling for ITER and its integration into the plasma control system is in progress [7].

Many facilities are now starting to work toward more integrated control as will be needed in ITER. Key actuators are likely to be oversubscribed, and algorithms for actuator sharing based on generalized, deviceindependent logic are being developed [88]. Integrated control with actuator sharing has been demonstrated on ASDEX Upgrade [72], [73], and TCV [74], [75]. In the example from TCV shown in Fig. 14, gyrotrons are used for simultaneous control of beta, and central q-profile, and switched to NTM control as needed.

With similar goals, DIII-D has implemented a generalized Off-Normal/Fault Response (ONFR) system to handle off-normal plasma events, and hardware faults [79]. Based on finite-state machine logic, the ONFR consists of multiple discrete operating states with rules for the transitions between states, and the rules may vary with the state. It provides the supervisory logic for recognition of off-normal conditions and appropriate actions to recover the discharge, change to an alternate mode of operation, or shut down the discharge. In a typical application, the ONFR system employs ECCD (continuous and pulsed) and forced island rotation by an applied $\mathrm{n}=1$ field to limit the growth of an $\mathrm{n}=1$ tearing mode, enabling a stable rampdown without disruption [92].

As the largest tokamak now in operation, JET faces many of the same issues as ITER. In preparation for the coming D-T campaign, JET has added controls to maintain the required operating point (including isotope ratio), carry out a controlled shutdown if the discharge is not evolving as planned, and trigger the disruption mitigation valve if a disruption is judged inevitable [76]. Work is in progress to ensure integration of these controllers with the rest of the control system [77]. The impact of central impurity accumulation on stable 
operation, including stable discharge termination, will be addressed by methods including improved detection of impurity accumulation, and optimization of ELM pacing by pellets and ICRH heating [94].

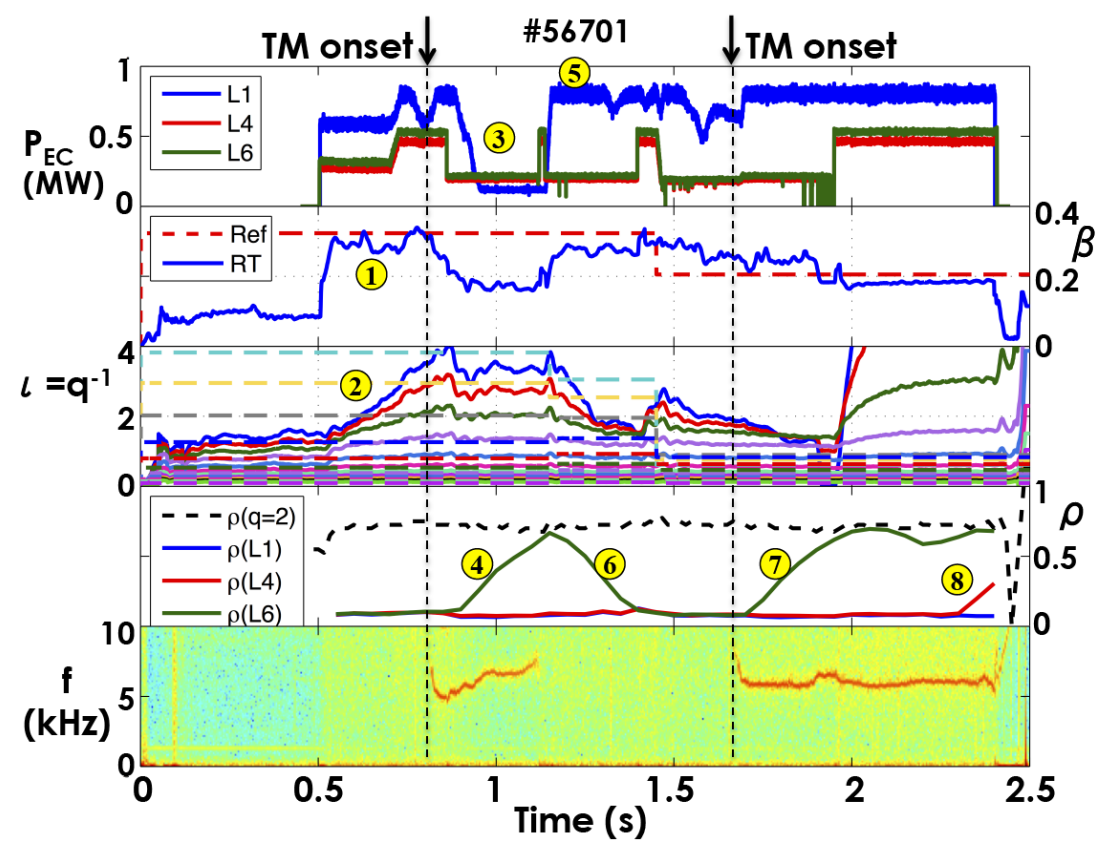

Fig. 14. TCV discharge showing integrated control with actuator sharing. $(1,2)$ During normal operation, central ECCD controls beta and q-profile. (3,4) After neoclassical tearing mode (NTM) onset, the heating power is reduced and one EC launcher is re-aimed to $q=2$. $(5,6)$ After stabilization of the NTM, heating power is raised and the launcher is returned to central current drive. (7,8) After onset of a second NTM, one EC launcheris re-aimed to $q=2$. The EC pulse ends as a second launcher is being targeted to $q=2$. [Reprinted from M. Kong, et al., Proc. 44th EPS Conf. on Plasma Physics, Europhysics Conf. Abstracts, Vol. $41 F$ (2017), paper P4.152.]

\section{DISCUSSION}

Many of the key elements of disruption-free tokamak operation have been demonstrated in present devices. The challenge now for present large and medium-size tokamaks is twofold. First, disruption prevention must move beyond proof-of principle experiments to become an integrated part of the facility's normal operation, in order to demonstrate robustly stable operation. Routine, integrated disruption prevention over a range of plasma conditions (preferably with diagnostics and actuators similar to those available in ITER) will enhance the reliability and consistency of operation of today's devices, build confidence in the ability of tokamaks to operate without disruptions, and drive the control engineering development that is necessary for extension to ITER and other future devices. Second, methods of warning and prevention of disruptions must move beyond empirical criteria to establish well-supported physics bases, in order to enable application of the methods in future devices with little or no empirical training. This work is vital in preparation for disruption-free operation of ITER.

Theory and simulations will continue to be crucial in the development of disruption-free operation. Ideal MHD models have been successful in predicting stability limits and the response to external magnetic perturbations, and kinetic models have extended these capabilities to accurately predict the stability of resistive wall modes. In future work, nonlinear, nonideal models should be further extended, in order to predict the onset, growth, and saturation of metastable or linearly unstable tearing modes, for example, and to guide methods of maintaining or restoring stability through EC power [80], [81] or applied magnetic perturbations [62], [63]. Modeling is essential in order to develop the physics understanding that is needed to extend present results in disruption warning and prevention to ITER. In addition, a key goal of this research should be to validate "reduced" models capable of predicting the discharge evolution and stability in real time.

Work supported by US DOE under DE-FC02-04ER54698, DE-SC0008520, DE-SC0016372, DE-FG0204ER54761，DE-AC52-07NA27344，DE-SC0015878，DE-SC0014264，DE-FG02-99ER54524，DE-FOA0001498, DE-AC02-09CH11466, DE-FC02-99ER54512, DE-SC0010720, DE-SC0010492. DIII-D data shown in this paper can be obtained in digital format by following the links at https://fusion.gat.com/global/D3D DMP. Disclaimers: This report was prepared as an account of work sponsored by an agency of the United States Government. Neither the United States Government nor any agency thereof, nor any of their employees, makes 
any warranty, express or implied, or assumes any legal liability or responsibility for the accuracy, completeness, or usefulness of any information, apparatus, product, or process disclosed, or represents that its use would not infringe privately owned rights. Reference herein to any specific commercial product, process, or service by trade name, trademark, manufacturer, or otherwise does not necessarily constitute or imply its endorsement, recommendation, or favoring by the United States Government or any agency thereof. This work has been carried out within the framework of the EUROfusion Consortium and has received funding from the Euratom research and training programme 2014-2018 under grant agreement No 633053. The views and opinions expressed herein do not necessarily reflect those of the European Commission. ITER is the Nuclear Facility INB no. 174. The views and opinions expressed herein do not necessarily reflect those of the ITER Organization. This publication is provided for scientific purposes only. Its contents should not be considered as commitments from the ITER Organization as a nuclear operator in the frame of the licensing process. This work was supported in part by the Swiss National Science Foundation.

\section{REFERENCES}

[1] LEHNEN, M., et al., "Plasma disruption management in ITER," Proc. 26th IAEA Fusion Energy Conference (Kyoto, 2016), IAEA, Vienna (2017), paper EX/P6-39.

[2] ITER ORGANIZATION, ITER Research Plan within the Staged Approach (Level III - Provisional Version), ITER Report ITR-18-003 (2018).

[3] LEHNEN, M., et al., Disruptions in ITER and strategies for their control and mitigation, Journal of Nuclear Materials 463 (2015) 39.

[4] DE VRIES, P.C., et al., Requirements for triggering the ITER disruption mitigation system, Fus. Sci. Technology 69 (2016) 471.

[5] BOOZER, A.H., Pivotal issues on relativistic electrons in ITER, Nucl. Fusion 58 (2018) 036006.

[6] KONOVAlOV, S., et al., Assessment of the Runaway Electron Energy Dissipation in ITER, Proc. 26th IAEA Fusion Energy Conference (Kyoto, 2016), IAEA, Vienna (2017), paper TH/7-1.

[7] RAUPP, G., et al., Preliminary exception handling analysis for the ITER plasma control system, Fusion Engineering and Design 123 (2017) 541.

[8] HUMPHREYS, D., et al., Novel aspects of control in ITER, Phys. Plasmas 22 (2015) 021806.

[9] PAUTASSO, G., et al., The ITER disruption mitigation trigger: developing its preliminary design, Nucl. Fusion 58 (2018) 036011.

[10] BUTTERY, R.J., et al., The influence of rotation on the $\beta_{\mathrm{N}}$ threshold for the $2 / 1$ neoclassical tearing mode in DIIID, Phys. Plas. 15 (2008) 056115.

[11] HENDER, T.C., et al., The role of MHD in causing impurity peaking in JET hybrid plasmas, Nucl. Fusion 56 (2016) 066002.

[12] TURCO, F., et al., The Causes of the Disruptive Tearing Instabilities of the ITER Baseline Scenario in DIII-D, Nuclear Fusion 58 (2018) 106043.

[13] FELICI, F., et al., Real-time model-based plasma state estimation, monitoring and integrated control in TCV, ASDEX Upgrade and ITER, Proc. 26th IAEA Fusion Energy Conference (Kyoto, 2016), IAEA, Vienna (2017), paper EX/P8-33.

[14] FELICI, F., et al., Real-time-capable prediction of temperature and density profiles in a tokamak using RAPTOR and a first-principle-based transport model, Nucl. Fusion 58 (2018) 096006.

[15] MAVKOV, B., et al., Experimental validation of a Lyapunov-based controller for the plasma safety factor and plasma pressure in the TCV tokamak, Nucl. Fusion 58 (2018) 056011.

[16] WEHNER, W., et al., Optimal current profile control for enhanced repeatability of L-mode and H-mode discharges in DIII-D, Fusion Engineering and Design 123 (2017) 513. 
[17] WEHNER, W.P, et al., "Physics-model-based real-time optimization for the development of steady-state scenarios at DIII-D," Proc. 27th IAEA Fusion Energy Conference (Ahmedabad, 2018), IAEA, Vienna (2019), paper EX/P639.

[18] LA HAYE, R.J., et al., Effect of thick blanket modules on neoclassical tearing mode locking in ITER, Nucl. Fusion 57 (2017) 014004.

[19] POLI, F.M., et al., Electron cyclotron power management for control of neoclassical tearing modes in the ITER baseline scenario, Nucl. Fusion 58 (2018) 016007.

[20] KIM, D., "Real-Time Control of Sawteeth and NTMs in TCV and ITER," Ph.D. Thesis, École Polytechnique Fédérale de Lausanne, Switzerland (2015) https://infoscience.epfl.ch/record/205040/files/EPFL TH6539.pdf.

[21] MARASCHEK, M., et al., Real-time control of MHD instabilities using ECCD in ASDEX Upgrade, Proc. 42nd EPS Conf. on Plasma Physics, Europhysics Conf. Abstracts, Vol. 39E (2015), paper P1.110.

[22] KASPAREK, W., et al., NTM stabilization by alternating O-point EC current drive using a high-power diplexer, Nucl. Fusion 56 (2016) 126001.

[23] CHAPMAN, I.T., et al., Empirical scaling of sawtooth period for onset of neoclassical tearing modes, Nucl. Fusion 50 (2010) 102001.

[24] CHAPMAN, I.T., et al., Power requirements for electron cyclotron current drive and ion cyclotron resonance heating for sawtooth control in ITER, Nucl. Fusion 53 (2013) 066001.

[25] GRAVES, J.P., et al., Sawtooth control in JET with ITER relevant low field side resonance ion cyclotron resonance heating and ITER-like wall, Plasma Phys. Control. Fusion 57 (2015) 014033.

[26] LERCHE, E., et al., Sawtooth pacing with on-axis ICRH modulation in JET-ILW, Nucl. Fusion 57 (2017) 036027.

[27] LANCTOT, M.J., et al., Impact of toroidal and poloidal mode spectra on the control of non-axisymmetric fields in tokamaks, Physics of Plasmas 24 (2017) 056117.

[28] SABBAGH, S.A., et al., Overview of physics results from the conclusive operation of the National Spherical Torus Experiment, Nuc. Fusion 53 (2013) 104007.

[29] HANSON, J.M., et al., Stability of DIII-D high-performance, negative central shear discharges, Nucl. Fusion 57 (2017) 056009.

[30] CLEMENT, M., et al., H2 optimal control techniques for resistive wall mode feedback in tokamaks, Nucl. Fusion 58 (2018) 046017.

[31] DE VRIES, P.C., et al., Scaling of the MHD perturbation amplitude required to trigger a disruption and predictions for ITER, Nucl. Fusion 56 (2016) 026007.

[32] SWEENEY, R., et al., Statistical analysis of $m / n=2 / 1$ locked and quasi-stationary modes with rotating precursors at DIII-D, Nucl. Fusion 57 (2017) 016019.

[33] SOZZI, C., et al., Early identification of disruption paths for prevention and avoidance, Proc. 27th IAEA Fusion Energy Conference (Ahmedabad, 2018), IAEA, Vienna (2019), paper EX/P1-22.

[34] GERHARDT, S.P., et al., Detection of disruptions in the high- $\beta$ spherical torus NSTX, Nucl. Fusion 53 (2013) 063021.

[35] DE VRIES, P.C., et al., Survey of disruption causes at JET, Nucl. Fusion 51 (2011) 053018.

[36] SABBAGH, S.A., et al., Disruption event characterization and forecasting in tokamaks, Proc. 27th IAEA Fusion Energy Conference (Ahmedabad, 2018), IAEA, Vienna (2019), paper EX/P6-26.

[37] BERKERY, J.W., et al., A reduced resistive wall mode kinetic stability model for disruption forecasting, Phys. Plasmas 24 (2017) 056103. 
[38] BERKERY, J.W., et al., Characterization and forecasting of global and tearing mode stability for tokamak disruption avoidance, Proc. 44th EPS Conf. on Plasma Physics, Europhysics Conf. Abstracts, Vol. 41F (2017), paper P1.138.

[39] MARASCHEK, M., et al., Path-oriented early reaction to approaching disruptions in ASDEX Upgrade and TCV in view of the future needs for ITER and DEMO Plasma Phys. Control. Fusion 60 (2018) 014047.

[40] GLASSER, A. S., KOLEMEN, E., and GLASSER, A. H., A Riccati solution for the ideal MHD plasma response with applications to real-time stability control, Physics of Plasmas 25, 032507 (2018).

[41] GLASSER, A. S., and KOLEMEN, E., A robust solution for the resistive MHD toroidal $\Delta^{\prime}$ matrix in near realtime, Physics of Plasmas 25, 082502 (2018).

[42] REIMERDES, H., et al., Measurement of the Resistive-Wall-Mode Stability in a Rotating Plasma Using Active MHD Spectroscopy, Phys. Rev. Lett. 93 (2004) 135002.

[43] TURCO, F., et al., Current density and rotation profiles govern the stability of the ITER baseline scenario in DIIID; predictive transport simulations capture the confinement degradation due to direct electron heating, Proc. 26th IAEA Fusion Energy Conference (Kyoto, 2016), IAEA, Vienna (2017), paper EX/P3-14.

[44] HANSON, J.M., et al., Understanding and controlling the ITER baseline plasma response, Proc. 45th EPS Conf. on Plasma Physics, Europhysics Conf. Abstracts, Vol. 42A (2018) paper P2.1110.

[45] RATTA, G.A., et al., An advanced disruption predictor for JET tested in a simulated real-time environment, Nucl. Fusion 50 (2010) 025005.

[46] VEGA, J., et al., Adaptive high learning rate probabilistic disruption predictors from scratch for the next generation of tokamaks, Nucl. Fusion 54 (2014) 123001.

[47] MURARI, A., et al., Adaptive predictors based on probabilistic SVM for real time disruption mitigation on JET, Nucl. Fusion 58 (2018) 056002.

[48] KLEIJWEGT, K, et al., Disruption prediction using machine learning algorithms for DIII-D, General Atomics Report GA-A28645, submitted to Nuclear Fusion (2018).

[49] OLOFSSON, K.E.J., et al., Event hazard function learning and survival analysis for tearing mode onset characterization, Plasma Phys. Control. Fusion 60 (2018) 084002.

[50] KATES-HARBECK, J., et al., Accelerating progress towards controlled fusion power via deep learning at the largest scale, to be submitted to Nature (2018).

[51] REA, C., et al., Initial Results of a Machine Learning-based Real Time Disruption Predictor on DIII-D, Proc. 45th EPS Conf. on Plasma Physics, Europhysics Conf. Abstracts, Vol. 42A (2018), paper P4.1076.

[52] GRANETZ, R.S., et al., Machine learning for disruption warning on ALCATOR C-MOD, DIII-D, and EAST tokamaks, Proc. 27th IAEA Fusion Energy Conference (Ahmedabad, 2018), IAEA, Vienna (2019), paper EX/P620 .

[53] CANNAS, B., et al, Automatic disruption classification in JET with the ITER-like wall, Plasma Phys. Control. Fusion 57 (2015) 125003.

[54] PAU, A., et al., A First Analysis of JET Plasma Profile-Based Indicators for Disruption Prediction and Avoidance, IEEE Trans. Plasma Science, 46 (2018) 2691.

[55] PARSONS, M., Interpretation of machine-learning-based disruption models for plasma control, Plasma Phys. Control. Fusion 59 (2017) 085001.

[56] PACCAGNELLA. R., et al., Entrainment of MHD modes in ASDEX Upgrade using rotating non-axisymmetric perturbation fields, Proc. 43rd EPS Conf. on Plasma Physics, Europhysics Conf. Abstracts, Vol. 40A (2016), paper P1.027. 
[57] OKABAYASHI, M., et al., Avoidance of tearing mode locking with electromagnetic torque introduced by feedback-based mode rotation control in DIII-D and RFX-mod, Nucl. Fusion 57 (2017) 016035.

[58] CHOI, W., et al., Feedforward and feedback control of locked mode phase and rotation in DIII-D with application to modulated ECCD experiments, Nucl. Fusion 58 (2018) 036022.

[59] JIN, HAI, et al., Locked mode unlocking by rotating resonant magnetic perturbations in J-TEXT tokamak, Plasma Phys. Control. Fusion 57 (2015) 104007.

[60] HU, QIMING and YU, Q., Suppressing magnetic island and accelerating its rotation by modulated resonant magnetic perturbation, Nucl. Fusion 56 (2016) 034001.

[61] OKABAYASHI, M., et al, Physics of Unlocked Tearing Modes and Disruption Avoidance by Feedback-based Electromagnetic Torque Injection, Proc. 26th IAEA Fusion Energy Conference (Kyoto, 2016), IAEA, Vienna (2017), paper EX/P3-16.

[62] YU, Q. et al., Suppressing magnetic island growth by resonant magnetic perturbation, Nucl. Fusion 58 (2018) 054003 .

[63] INOUE, S., et al., Dependence of mode locking/unlocking on frequency of rotating 3D field and its experimental application for active locked mode avoidance, Proc. 27th IAEA Fusion Energy Conference (Ahmedabad, 2018), IAEA, Vienna (2019), paper TH/P5-24.

[64] ESPOSITO, B., et al., Disruption avoidance by means of electron cyclotron waves, Plasma Phys. Control. Fusion 53 (2011) 124035.

[65] GRANUCCI, G., et al., Stable Operation at Disruptive Limits by Means of EC at ASDEX Upgrade, Proc. 42nd EPS Conf. on Plasma Physics, Europhysics Conf. Abstracts, Vol. 39E (2015) paper P1.108.

[66] VOLPE, F.A., et al., Avoiding tokamak disruptions by applying static magnetic fields that align locked modes with stabilizing wave-driven currents, Phys. Rev. Letters 115 (2015) 175002.

[67] DE VRIES, P.C., et al., Multi-machine analysis of termination scenarios with comparison to simulations of controlled shutdown of ITER discharges, Nucl. Fusion 58 (2018) 026019.

[68] BARR, J.L., et al., Fast ITER-relevant low-disruptivity rampdowns in DIII-D and EAST, Proc. 27th IAEA Fusion Energy Conference (Ahmedabad, 2018), IAEA, Vienna (2019), paper EX/P6-21.

[69] WOO, M.H., et al., Implementation of the forced landing scheme under off-normal events in KSTAR, Fusion Engineering and Design 128 (2018) 168.

[70] HOBIRK, J., et al., Analysis of plasma termination in the JET hybrid scenario, Nucl. Fusion 58 (2018) 076027.

[71] DE LA LUNA, E., et al., Impact of ELM control in JET experiments on H-mode terminations with/without current ramp-down and implications for ITER, Proc. 27th IAEA Fusion Energy Conference (Ahmedabad, 2018), IAEA, Vienna (2019), paper EX/2-1.

[72] RAPSON, C.J., et al., Experiments on actuator management and integrated control at ASDEX Upgrade, Fusion Engineering and Design 123 (2017) 603.

[73] KUDLACEK, O., et al., "Actuator management development on ASDEX-Upgrade," 30th Symposium on Fusion Technology (SOFT 2018), paper P2.033, to be submitted to Fusion Engineering and Design.

[74] KONG, M., et al., Real-time control of neoclassical tearing modes and its integration with multiple controllers in the TCV Tokamak, Proc. 44th EPS Conf. on Plasma Physics, Europhysics Conf. Abstracts, Vol. 41F (2017) paper P4.152.

[75] KONG, M., et al., Control of NTMs and integrated multi-actuator control on TCV, Proc. 27th IAEA Fusion Energy Conference (Ahmedabad, 2018), IAEA, Vienna (2019), paper EX/P1-25.

[76] LENNHOLM, M., et al., Real time control developments at JET in preparation for deuterium-tritium operation, Fusion Engineering and Design 123 (2017) 535. 
[77] JOFFRIN, E., et al., Overview of the JET preparation for Deuterium-Tritium Operation, Proc. 27th IAEA Fusion Energy Conference (Ahmedabad, 2018), IAEA, Vienna (2019), paper OV/1-3.

[78] SHEIKH, U.A., et al.., Disruption avoidance through the prevention of NTM destabilization in TCV, Plasma Nuclear Fusion 58 (2018) 106026.

[79] EIDIETIS, N.W., et al., Implementing a finite-state off-normal and fault response system for disruption avoidance in tokamaks, Nucl. Fusion 58 (2018) 056023.

[80] PRATT, J., et al., Early evolution of electron cyclotron driven current during suppression of tearing modes in a circular tokamak, Phys. Plasmas 23 (2016) 102507.

[81] LAZZARO, E, et al., Physics conditions for robust control of tearing modes in a rotating tokamak plasma, Plasma Phys. Control. Fusion 60 (2018) 104044.

[82] DE VRIES, P.C., et al., The influence of an ITER-like wall on disruptions at JET, Phys. Plasmas 21 (2014) 056101 .

[83] NEU, R., et al., Overview on plasma operation with a full tungsten wall in ASDEX Upgrade, J. Nucl. Mater 438 (2013) S34.

[84] GERASIMOV, S.N., et al., Overview of Disruptions with JET-ILW, Proc. 27th IAEA Fusion Energy Conference (Ahmedabad, 2018), IAEA, Vienna (2019), paper EX/P1-24.

[85] MALJAARS, E., et al, Profile control simulations and experiments on TCV: a controller test environment and results using a model-based predictive controller, Nucl. Fusion 57 (2017) 126063.

[86] KIM, D., et al., An active feedback plasma profile control approach applied to TCV plasmas and perspectives toward ITER 43rd EPS Conf. on Plasma Physics, Europhysics Conf. Abstracts, Vol. 40A (2016), paper P4.069.

[87] TEPLUKHINA, A.A., et al. Simulation of profile evolution from ramp-up to ramp-down and optimization of tokamak plasma termination with the RAPTOR code. Plasma Phys. Control. Fusion, 59 (2017) 124004.

[88] MALJAARS, E. and FELICI, F., Actuator allocation for integrated control in tokamaks: architectural design and a mixed-integer programming algorithm, Fusion Engineering and Design 122 (2017) 94.

[89] YU, Q., et al., NTM Excitation by Sawtooth Crashes and the Suppression of their Onset by Resonant Magnetic Perturbation, Proc. 27th IAEA Fusion Energy Conference (Ahmedabad, 2018), IAEA, Vienna (2019), paper TH/P5-19.

[90] OKABAYASHI, M., et al., Critical Processes of Tearing Mode Entrainment in the Presence of a Static Error Field, Proc. 27th IAEA Fusion Energy Conference (Ahmedabad, 2018), IAEA, Vienna (2019), paper EX/P6-25.

[91] POLI, F.M., et al., The Plasma Current Ramp-Down in ITER: Physics Constraints on Control, Proc. 27th IAEA Fusion Energy Conference (Ahmedabad, 2018), IAEA, Vienna (2019), paper EX/P7-27.

[92] EIDIETIS, N.W., et al., Implementing a Finite-State Off-Normal and Fault Response System for Robust Disruption Avoidance in Tokamaks, Proc. 27th IAEA Fusion Energy Conference (Ahmedabad, 2018), IAEA, Vienna (2019), paper EX/P6-22.

[93] REA, C., et al., Disruption prediction investigations using Machine Learning tools on DIII-D and Alcator C-Mod, Plasma Phys. Control. Fusion 60 (2018) 084004.

[94] GARZOTTI, L., et al., Scenario Development for DT Operation at JET, Proc. 27th IAEA Fusion Energy Conference (Ahmedabad, 2018), IAEA, Vienna (2019), paper EX/3-6.

[95] VU, T., et al., Plasma internal profile control using IDA-PBC: Application to TCV, Fusion Engineering and Design 123 (2017) 624.

[96] ANGIONI, C., et al., Tungsten transport in JET H-mode plasmas in hybrid scenario, experimental observations and modelling, Nucl. Fusion 54 (2014), 083028. 
[95][97] RIMINI, F.G., et al., The development of safe high current operation in JET-ILW, Fusion Engineering and Design 96-97 (2015) 165. 